\title{
Circular dichroism from Fano resonances in planar chiral oligomers
}

\author{
Ben Hopkins ${ }^{*, a}$, Alexander N. Poddubny ${ }^{b, c}$, Andrey E. Miroshnichenko ${ }^{a}$ and \\ Yuri S. Kivshar ${ }^{a, c}$ \\ ${ }^{a}$ Nonlinear Physics Centre, Australian National University, Canberra, ACT 2601, Australia; \\ ${ }^{b}$ Ioffe Physical-Technical Institute of the Russian Academy of Sciences, St. Petersburg 194021, \\ Russia; \\ ${ }^{c}$ National Research University for Information Technology, Mechanics and Optics, St. \\ Petersburg 197101, Russia
}

\begin{abstract}
Here we present a general approach for describing the physics of Fano resonances in nanoparticle oligomers. It is shown that the interference of nonorthogonal collective eigenmodes is a sufficient condition to produce Fano resonances. We then show that such nonorthogonality between eigenmodes also permits the existence of a new form circular dichroism in the absorption and scattering cross-sections, even when circular dichroism is forbidden in the extinction cross-section.
\end{abstract}

Keywords: Metasurface, Chiral, Oligomer, Fano, Plasmonic, Rotational symmetry

\section{INTRODUCTION}

The widespread desire for strong optical interactions with chiral molecules and proteins, present in many forms of biologicals and pharmaceuticals, has made nanoscale chiral materials and particles some of most imminentlyapplicable devices in the broader field of optical metamaterials. At the same time, a growing proportion of metamaterial research is being devoted toward planar metasurfaces; ultrathin functional surfaces that are unhindered by the structural design constraints of three-dimensional fabrication. Here we present a new form of circular dichroism produced by switching the loss mechanism of a plasmonic nanostructure between far-field radiation and near-field material absorption, ${ }^{1}$ both of which are key (and easily observable) forms of chiral response. Importantly, this effect is able to exist in planar systems and we show it is fundamentally linked to eigenmode interference, a consequence of which is that it can be amplified through Fano resonances. Using this amplification, the relative magnitude of such circular dichroism in absorption and scattering cross sections can easily be an order of magnitude larger than that typically reported in the extinction of three-dimensional nanostructures. This work subsequently projects both metasurfaces and planar nanoparticle arrays into the realm of chiral optical devices, opening up a host of new opportunities to capitalize on their resonant mechanics and implicit design freedom. In addition to this, we introduce a new approach to circular dichroism starting from a ground-up description of its origin in terms of the current distributions that are induced by circularly polarized plane waves. This analysis allows us to deduce why the circular dichroism can exist in planar structures in spite of reciprocity. We also focus on rotationally-symmetric structures because they exhibit absorption and scattering invariance to linear polarisations, ${ }^{2}$ a practicality which is necessary to ensure the independence of a cross-section to the phase between LCP and RCP light. Under this constraint we are able to analytically derive design principles to intuitively identify and demonstrate that planar chiral oligomers are an obvious nanoparticle geometry that exhibits Fano-resonance-enhanced circular dichroism.

\footnotetext{
*E-mail: ben.hopkins@anu.edu.au
} 


\section{FANO RESONANCES IN SYMMETRIC NANOPARTICLE OLIGOMERS}

The modes of plasmonic nanostructures are typically understood based on their electric dipole moments or current distributions. Therefore, the eigenmodes of plasmonic nanostructures can be calculated as the harmonic current distributions or dipole moment profiles that are preserved under coupling. More formally, the relationship between the current distribution $(\mathbf{J}(\boldsymbol{x}))$ in an arbitrary compact scatterer, and an externally-applied incident electric field distribution $\left(\mathbf{E}_{0}(\boldsymbol{x})\right)$ can be formulated in terms of dyadic Green's function coupling. ${ }^{1,3}$

$$
i \omega \mathbf{E}_{0}(\boldsymbol{x})=-\left[\overline{\boldsymbol{\epsilon}}(\boldsymbol{x})-\epsilon_{0}\right]^{-1} \mathbf{J}(\boldsymbol{x})+\int_{V_{s}} \overline{\mathcal{G}}\left(\boldsymbol{x}, \boldsymbol{x}^{\prime}\right) \mathbf{J}\left(\boldsymbol{x}^{\prime}\right) \mathrm{d}^{3} x^{\prime} .
$$

where the volume $V_{s}$ is the volume of the compact scatterer and $\overline{\mathcal{G}}$ is a generalized dyadic Green's function, written in terms of the source dyadic $(\overline{\mathbf{L}})$ and the free space Green's function

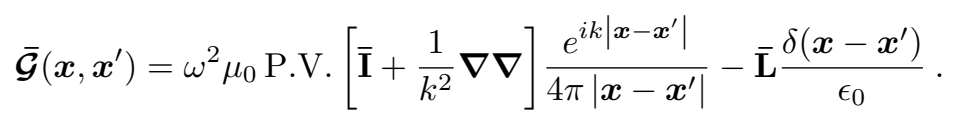

Here, $\omega$ is the angular frequency of light and P.V. implies a principle value exclusion of $\boldsymbol{x}=\boldsymbol{x}^{\prime}$ when performing the integration in Eq. 1. For ease of notation, we have also defined a tensor permittivity $(\bar{\epsilon})$ to describe both the conductivity $(\overline{\boldsymbol{\sigma}})$ and electric susceptibility $(\bar{\chi})$ of the material

$$
\overline{\boldsymbol{\epsilon}} \equiv(\bar{\chi}+1) \epsilon_{0}-\frac{\overline{\boldsymbol{\sigma}}}{i \omega} \quad \Rightarrow \quad \mathbf{J}(\boldsymbol{x})=-i \omega\left[\overline{\boldsymbol{\epsilon}}(\boldsymbol{x})-\epsilon_{0}\right] \mathbf{E}(\boldsymbol{x})
$$

For the discussion here, we can consider the relationship above in terms of an interaction operator $\hat{M}$, which links the incident field distribution, $\left|E_{0}\right\rangle$, to the induced dipole moments, $|p\rangle$.

$$
\left|E_{0}\right\rangle=\hat{M}|p\rangle
$$

The use of the 'bra-ket' notation for the incident field and dipole moments is simply to emphasize that each state is the concatenation of all the dipole-moment vectors in the given system. The existence of radiative and/or dissipative losses then guarantees that the interaction matrix is non-Hermitian (i.e., having complex eigenvalues). This therefore permits the system's eigenmodes to be nonorthogonal. Subsequently we can expect the response of a system with multiple excited eigenmodes to exhibit modal interference. We can illustrate this point by considering an incident field written as a linear combination of two nondegenerate eigenmodes,

$$
\begin{aligned}
& \left|E_{0}\right\rangle=a_{1}\left|\nu_{1}\right\rangle+a_{2}\left|\nu_{2}\right\rangle \\
& \Rightarrow|p\rangle=a_{1} \lambda_{1}\left|\nu_{1}\right\rangle+a_{2} \lambda_{2}\left|\nu_{2}\right\rangle .
\end{aligned}
$$

Here the coefficients of each eigenmode in the induced dipole moment solution (i.e., the $a_{i} \lambda_{i}$ terms) represent the magnitude of excitation for each eigenmode. Now the extinction cross section is proportional to the imaginary component of the projection of the dipole moments onto the incident field

$$
\sigma_{e} \propto \operatorname{Im}\left\{\left\langle E_{0} \mid p\right\rangle\right\}
$$

Subsequently, in this extinction expression, the nonorthogonality of the eigenmodes will create cross terms from eigenmodes projected onto each other

$$
\begin{aligned}
\left\langle E_{0} \mid p\right\rangle & =\left(a _ { 1 } ^ { * } \left\langle\nu_{1}\left|+a_{2}^{*}\left\langle\nu_{2}\right|\right)\left(a_{1} \lambda_{1}\left|\nu_{1}\right\rangle+a_{2} \lambda_{2}\left|\nu_{2}\right\rangle\right)\right.\right. \\
& =\underbrace{\left|a_{1}\right|^{2} \lambda_{1}\left|\nu_{1}\right|^{2}+\left|a_{2}\right|^{2} \lambda_{2}\left|\nu_{2}\right|^{2}}_{\text {direct terms }}+\underbrace{a_{1}^{*} a_{2} \lambda_{2}\left\langle\nu_{1} \mid \nu_{2}\right\rangle+a_{2}^{*} a_{1} \lambda_{1}\left\langle\nu_{2} \mid \nu_{1}\right\rangle}_{\text {interference terms }} .
\end{aligned}
$$

In this expression, each of the "direct terms" represents the extinction in the case where the incident field has been structured to consist of only the one given eigenmode and therefore such direct terms can only produce a positive contribution to the extinction (lest energy conservation is broken). On the other hand, the "interference 


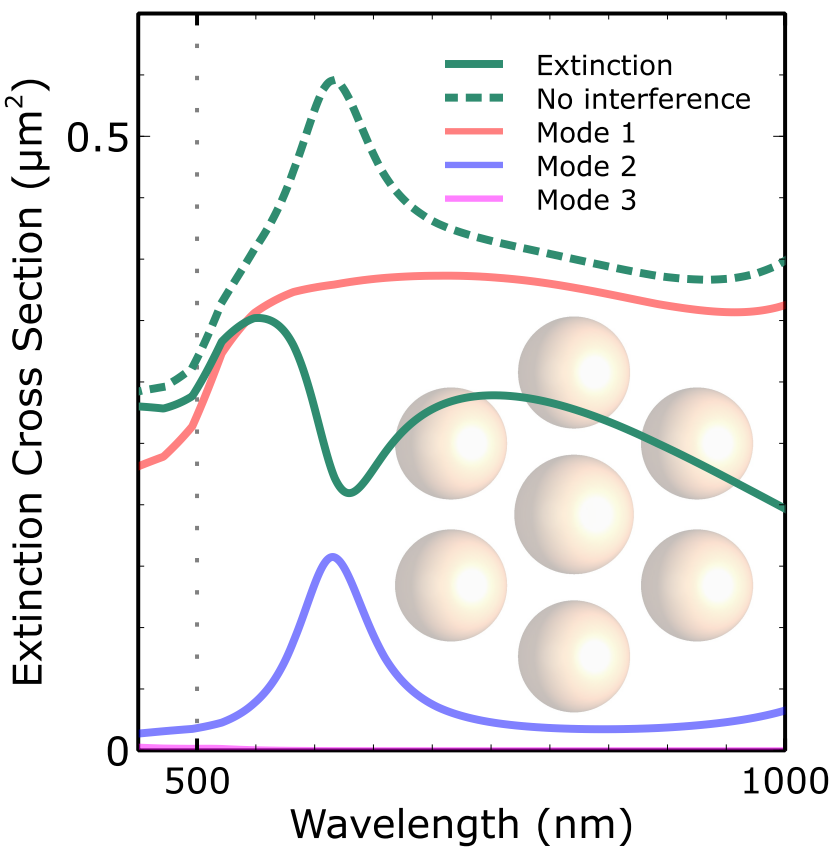

Figure 1. The simulated extinction spectrum of a gold nanosphere heptamer under normal plane wave excitation and its decomposition into contributions from three modes. The interference between the modes can be seen to be responsible for the Fano resonance.

terms" can be negative (i.e., destructive interference). Such interference can be seen in Figure 1 for a heptamer oligomer constructed of gold nanospheres under normal incidence (plane wave) excitation. In this figure, we can see that it is two bright modes that are interfering to produce the Fano resonance in extinction. In other words this demonstrates that dark modes do not need to play a role in producing a Fano resonance. Further, in Ref. $^{4}$, this approach is generalized by proving that the number of eigenmodes that can be excited in an arbitrary oligomer is set by symmetry and not the number of particles. Specifically it is shown that there will be two excitable eigenmodes per symmetric ring of particles and one further excitable eigenmode that can be created by adding a central particle.

\section{CIRCULAR DICHROISM IN PLANAR CHIRAL OLIGOMERS}

When a structure has a mirror plane perpendicular to the propagation direction of a plane wave, it is well-known that reciprocity is a sufficient condition to prevent any difference in the extinction cross-section between leftand right-handed polarizations. Such a difference in extinction cross-sections is commonly referred to as circular dichroism. However, in recent work, ${ }^{1}$ we showed that the existence of nonorthogonal eigenmodes permits the existence of circular dichroism in the absorption and scattering cross-sections of nanostructures that are both planar and chiral (in two dimensions). In other words, we should be able to observe significant circular dichroism in absorption and scattering cross-sections when there is a large eigenmode overlap, a situation which we know 
exists at a Fano resonance. Therefore, to produce circular dichroism in absorption, we can take a structure known to produce Fano resonances and alter it to be planar chiral. As such we can take a gold heptamer, much like that in Fig. 1 and then alter the nanoparticles in the outer ring to make it both planar chiral, while preserving $C_{n h}$ symmetry. This is what we have done for Fig. 2 . The choice of parameters was chosen so that the central particle resonance is overlapped with that of the outer ring. ${ }^{5}$ The outer ring in Fig 2 is a planar chiral

\section{Cross Sections $\left(\mu \mathrm{m}^{2}\right)$}

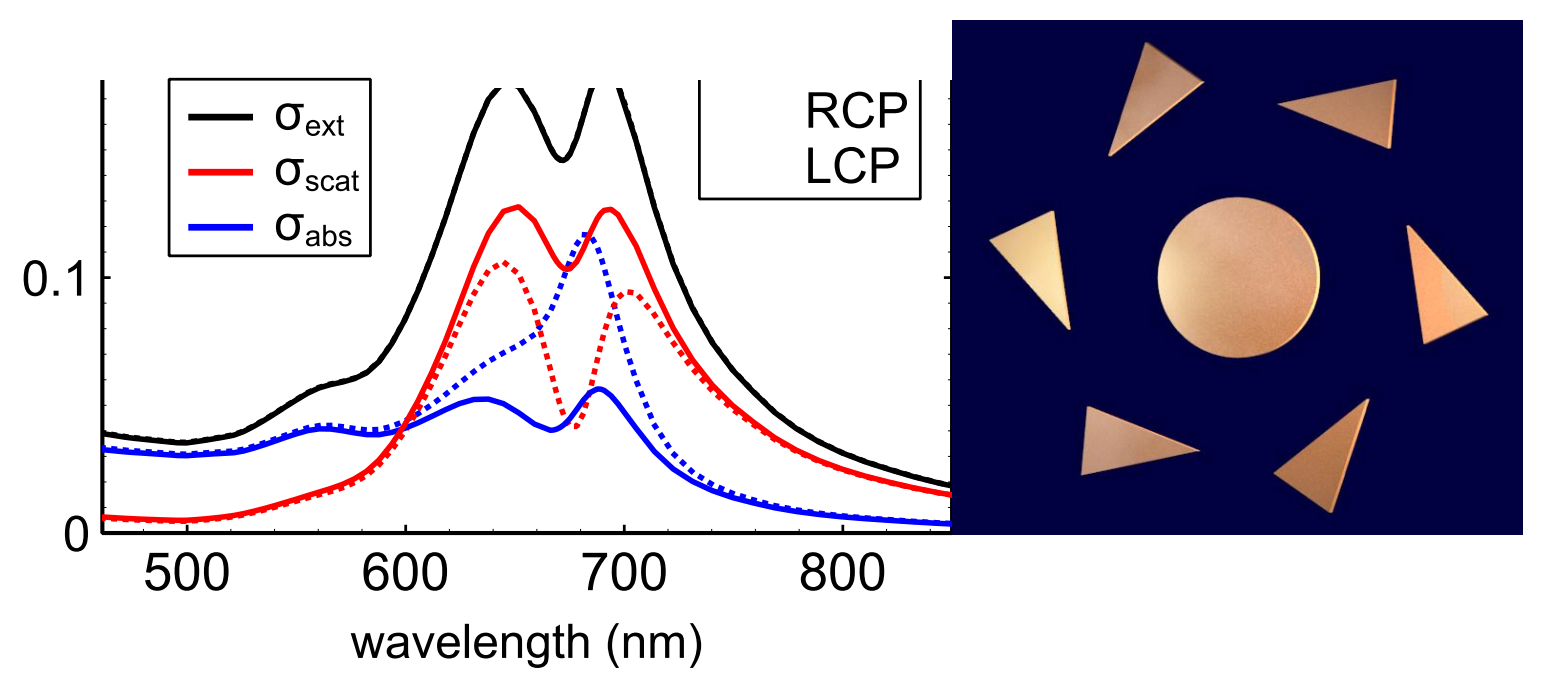

Figure 2. Simulations demonstrating the role of interference for inducing circular dichroism in the absorption cross-section of a planar chiral heptamer with $C_{6 h}$ symmetry. We observe the creation of significant circular dichroism in absorption in the vicinity of the Fano resonance, as is predicted given it corresponds to high modal overlap. The calculation parameters are given in text.

structure, and it will therefore experience different current distributions in response to LCP and RCP plane waves, but in isolation it does not experience significant circular dichroism because it doesn't exhibit a Fano resonance (See Ref. ${ }^{1}$ ). However the collective oligomer structure in Fig. 2 does exhibit a Fano resonance, and can thereby produce significant circular dichroism in absorption and scattering. The extent of the circular dichroism is, in fact, sufficient to swap the dominance of scattering and absorption cross-sections using polarization. This therefore supports the derivations in Ref. ${ }^{1}$ that nonreciprocal circular dichroism in absorption is an interference effect. In Fig. 2, all nanoparticles are gold and are $20 \mathrm{~nm}$ thick, the central disk has a diameter of 140nm and each triangular nanoparticle has major and minor axes of $100 \mathrm{~nm}$ and $60 \mathrm{~nm}$ (respectively). The triangular nanoparticles have been placed at a radius of $170 \mathrm{~nm}$ away from the center of the disk and the major axis is oriented $65^{\circ}$ off the radial vector. LCP and RCP are defined relative to a vector pointing out of the page. All simulations were performed using CST Microwave Studio and gold data was taken from Johnson and Christy. ${ }^{6}$ 


\section{CONCLUSIONS}

We have presented the results of a new approach for simplifying and understanding the response of rotationallysymmetric nanoparticle oligomers in terms of modal interference. This approach offers a platform on which to analyze and design strongly coupled nanoparticle systems, allowing the utilization of modal interference to engineer both chiral and polarization-invariant scattering behavior.

\section{REFERENCES}

1. B. Hopkins, A. N. Poddubny, A. E. Miroshnichenko, and Y. S. Kivshar, "Circular dichroism induced by fano resonances in planar chiral oligomers," arXiv , p. 1412.1120, 2014.

2. B. Hopkins, W. Liu, A. E. Miroshnichenko, and Y. S. Kivshar, "Optically isotropic responses induced by discrete rotational symmetry of nanoparticle clusters," Nanoscale 5, pp. 6395-6403, 2013.

3. A. D. Yaghjian, "Electric dyadic greens functions in the source region," Proc. IEEE 68(2), pp. 248-263, 1980.

4. B. Hopkins, A. N. Poddubny, A. E. Miroshnichenko, and Y. S. Kivshar, "Revisiting the physics of fano resonances for nanoparticle oligomers," Phys. Rev. A 88, p. 053819, 2013.

5. A. E. Miroshnichenko and Y. S. Kivshar, "Fano resonances in all-dielectric oligomers," Nano Lett. 12, pp. 6459-6463, 2012.

6. P. B. Johnson and R. W. Christy, "Optical constants of the noble metals," Phys. Rev. B 6, p. 4370, 1972. 